\title{
A CONEXÃO dA METOdISTA A INTERNET: A maIOR REDE do planeta
}

\author{
Maristela Dourado Rocha
}

\begin{abstract}
Resumo
Este trabalho visa um apanhado sobre a Internet: a rede que liga um computador a outro, estando localizado em qualquer parte do mundo, com o objetivo de trocar informações, conhecimentos, acervos e arquivos como também, do acesso à Internet adquirido pelo Instituto Metodista de Ensino Superior, a primeira instituição acadêmica do Grande $\mathrm{ABC}$ a se conectar mundialmente.
\end{abstract}

\section{Palavras-Chave}

Internet - Rede de computador - Informática

\section{A INTERNET}

A história da Internet começa com a pesquisa de técnicas para interconectar sistemas de computadores em 1960 nos EUA. Época da Guerra Fria, os norte americanos buscavam meios de se defender de um suposto iminente ataque russo. A rede de computadores serviria para disseminar informações de forma instantânea para todo o país. Esta rede para defesa dos EUA foi concebida de forma que se parte dela fosse destruída, o resto funcionaria.

Em 1969, surge a ARPANET, entidade que financia pesquisa em redes comutadas por pacotes. Nos primeiros quatro nós da Arpanet foram conectados os principais órgãos governamentais dos EUA e as maiores instituições de pesquisa científica.

Em 1978, surge o nome INTERNET, que passa a ser administrado pela DARPA. Em 1981, começam a operar a Bitnet e a CSNET. O protocolo TCP/IP se torna de domínio público para comunicação na Arpanet durante 1982. Os endereços de localização como são conhecidos hoje são introduzidos em 1984.

Um consórcio entre IBM, MCI e MERIT GRANT investe US\$ 14 milhões para administrar a rede durante o ano de 1987.

Em 1989, o backbone NSFNet chega à velocidade de 1,5 Mbp. E durante o ano de 1991, o uso comercial da Internet começa a se tornar viável.

A maior velocidade da rede de $45 \mathrm{Mbps}$, é inaugurada em 1992. Em 1995 começam a trafegar pela rede aplicações em áudio e vídeo digitais.

Para a passagem do século, há expectativa de que as aplicações multimídia sejam plenamente interativas na megarrede, com uso comercial e individual plenos. 


\section{O QUE VEM A SER A INTERNET?}

A Internet é uma super-rede de computadores capaz de interligar numa teia digital as residências, empresas e o comércio do mundo inteiro. Misto de correio instantâneo, já atinge 146 países e vem sendo usada por um número estimado em 30 milhões de pessoas. Calcula-se que até o final do século a mãe de todas as redes terá 100 milhões de usuários.

Simples de usar e barata, ela traz consigo a promessa de mudança radical no mundo dos negócios e a expectativa de um choque de modernidade na vida de seus usuários. A Internet é ao mesmo tempo uma aldeia eletrônica e um campus internacional, onde toda informação se organiza progressivamente em uma imensa biblioteca virtual. Trata-se de um gigantesco banco de ensaio para desenvolvimento de redes abertas cobrindo o conjunto do planeta.

Pessoas que vivem em regiões cuja distância chega aos milhões de km se comunicam sem nunca terem se visto, e há informações disponíveis $24 \mathrm{~h}$ por dia em centenas de lugares.

Surge a democratização da informação.

Profissionais liberais, estudantes, utilizamse dela para ter conhecimentos de outras culturas, ou mesmo ter informações específicas como novos estudos sobre aids, etc.

Os bibliotecários podem venerar a Internet pois ela possui avançadas ferramentas de pesquisa de documentos e do acesso quase instantâneo a catálogos e arquivos das principais bibliotecas e centros de documentação de todo o mundo, havendo no mínimo catálogos de 500 bibliotecas e diariamente mais são incluídas.

\section{COMO SE CONECTAR À MAIOR DAS REDES}

Primeiramente é necessário ter um computador, uma linha telefônica e um modem.

Para conseguir acesso comercial à Internet no Brasil é necessário contactar a EMBRATEL e a RNP - Rede Nacional de Pesquisas. A Embratel é uma das provedoras de serviços da megarrede, junto com as operadoras estaduais e empresas cadastradas. A RNP, além de provedora, tem a responsabilidade de definir os endereços dos novos usuários brasileiros.

A Embratel está prestando serviços nas modalidades UUCP(Unix to Unix Copy Program), IP discado, IP dedicado e Info Serv. Até Maio. o serviço continuou a ser prestado sob a forma de projeto piloto aos 250 usuários escolhidos pela estatal.

As pessoas que têm acesso à Internet através de uma organização, como uma universidade ou uma grande empresa, não precisam se preocupar com a forma como irão utilizá-la. Sua comunicação com pessoas de todo o mundo e o acesso à maioria dos recursos de informação não serão discriminados como ligações interurbanas em uma conta telefônica, porque as linhas alugadas ou as ligações da rede já estão pagas. Para esses usuários, é como se eles tivessem uma linha Wats sem limites.

O acesso para quem dispõe de um computador e de um modem é feito basicamente através de chamadas telefônicas locais com um servidor de computador.

As empresas cobram uma taxa fixa em torno de US\$ 20 mensalmente pela utilização ilimitada do correio eletrônico.

Mas pessoas que estão localizadas em lugares distantes, tem que pagar pelas chamadas a longa distância, caríssimas para enviar ou receber mensagens de correio eletrônico.

Com relação ao equipamento para conexão à Internet é necessário:

- configurar software de comunicação com os parâmetros:

emulação de terminal TTY
7 bits
paridade par
1 bit de stop ( 7 e 1$)$

Através do modem acessar RENPAC pelos n. 07878228 (para velocidade de transmissão de dados de 9600 bps - bits por segundo) ou 078 78224 (para 2400 bps)

Aguardar a resposta e logo após digitar 0724021050400 e teclar enter.

Preencher os campos com os dados e 
aguardar.

\section{ENDEREÇO ELETRÔNICO}

Para participar, cada usuário deve ter um endereço eletrônico (E-MAIL) ou username. O username normalmente apresenta primeiro um resumo do nome, à esquerda de um \&. Depois vem o nome do computador e o tipo de entidade a que o usuário pertence. Estes códigos são definidos por técnicos da entidade à qual está filiado e não permite mudança.

Ex. scdmsqui \& fox. cce.usp-br. Significa que o usuário Squirra tem uma caixa postal eletrônica no computador chamado fox, localizado no centro de computação eletrônica da USP. Termina com o código do país: br Brasil. Poderia trazer ainda a categoria do serviço: Edu (educacional), gov (governamental), mil (militar), com (comercial), org (organização não comercial) e net para redes.

O usuário recebe ainda um código de acesso que é pessoal e pode ser alterado.

Como fazer:

- Obter endereço eletrônico em companhias de grande porte, em alguma universidade, que tenha acesso Internet.

- pode ser feito através de terminais de vídeo desta instituição.

- modems - levar em consideração a taxa de transmissão de dados. Parte de 1200, 2400, $4800,9600,14400,19200$ e pode chegar a $38.400 \mathrm{bps}$ (bits por segundo).

- padrões de Data Bits, Paridade e Stop Bits. Padrão mundial de Data bits 8 .

\section{QUANTO CUSTA USAR A INTERNET?}

\section{SERVIÇO ALTERNEX}

Inscrição R \$ 10,00

Tempo de uso: Seg. à sexta - 8 às 20 hs sábado - 8 às 14 hs

Primeira hora do mês: R \$ 10,00

Segunda " " " : R $\$ 6,00$

Terceira " " " : $\mathrm{R} \$ 4,00$
Seg. à sexta $-20 \mathrm{hs}$ às $8 \mathrm{hs}$

Sábado e domingo - 14 hs em diante

Primeira hora do mês: $\mathrm{R} \$ 5,00$

Segunda “" " " : $\mathrm{R} \$ 3,00$

Terceira " " " : $\mathrm{R} \$ 2,00$

TARIFAS RENPAC : R \$ 0,03 min- R \$ 1.92 hora TRÁFEGO DE DADOS: R\$ 0,0017 por segmento (grupo 64 caracteres) em SP

ex. página de texto de 55 linhas de 64 caracteres $\mathrm{R} \$ 0,10$

dez páginas de texto - $\mathrm{R} \$ 1,00$

\section{SERVIÇOS QUE ELA OFERECE}

Na Internet é possível encontrar inúmeros serviços, softwares e banco de dados sobre diversas áreas.

\section{TURISMO}

É possível conhecer por fotos todo os roteiros da viagem dos sonhos. A viagem pode acabar por ser real.

Permite fazer reserva da tela do computador, ver fotos de lugares paradisíacos, comprar ingressos da Broadway antes de sair, ver o mapa e cardápios de alguns restaurantes para o destino que se quer ir e solicitar folhetos de turismo.

No Brasil, este tipo de serviço é incipiente, mas se há interesse da pessoa física, a Alternex pode ajudar.

Deve-se levar em conta as tarifas para não salgar a conta telefônica.

Através de protocolos de comunicação SLIP ou PPP, instala-se um programa chamado MOSAIC ou o NETSCAPE. Daí pode-se navegar pela WWW (WORLD WIDE WEB), rede de comunicação multimídia da Internet. Pelo W3 trafegam imagens, sons e documentos.

\section{CIÊNCIA} Internet.

Coisas inusitadas também aparecem na

Um homem condenado à morte nos EUA, 
decidiu doar seu corpo à ciência. A Universidade de Colorado congelou seu corpo e o cortou em 1871 fatias transversais de 1 milímetro que foram fotografadas. O corpo foi tomografado e feito ressonância magnética.

Esse feito ficou conhecido pelos usuários de o "Homem invisível".

Algumas imagens podem ser capturadas gratuitamente na Internet. Todos os dados são vendidos por US\$2000.

Endereço: http://www.nlm.nih.gov/ extramural resource. dir/visible human.htlm.

A Univ. de ULM na Alemanha, colocou na rede um laboratório virtual. O objetivo é ampliar o acesso de pesquisadores aos equipamentos de alta tecnologia disponíveis no laboratório de química. A USP também está no projeto.

\section{ENTRETENIMENTO}

$\mathrm{Na}$ Internet há um fórum de discussões sobre o trio de rap Beastie boys, numa linha exclusiva para fãs. É possível ouvir faixas de discos, imagens de vídeo, fotografias, discografia, etc.

O filme Batman está de volta e seu prélançamento pode ser conferido na Internet.

Quem tem acesso ao WWW, pode ver trailer, bastidores, fotos, etc. line.

A Internet já virou também sex-shop on-

Produtos são vendidos apenas fornecendo o número do cartão de crédito. Desde vibradores a bonecas. Mas não é só isso. Há grupos de discussões sobre diversos assuntos ligados a sexo. Desde casamentos entre descasados e solteiros até formas de fazer sexo.

O sexo virtual também tem virado mania. Em San Diego, Califórnia, há o Virtual Connections, o primeiro peep-show (show erótico em que o cliente observa a modelo por um visor). É só discar o n. da empresa e a imagem das modelos aparece. Elas conversam, fazem strip, tudo pelo computador.

\section{A INTERNET NO BRASIL}

Em 1988, já existiam conexões internacionais de redes não-comerciais com o exterior (Fapesp em SP, LNCC e UFRJ no RJ), mas ainda era utilizada somente a tecnologia BITNET (Because it's time Network), implementada basicamente em computadores IBM de grande porte, transmitindo apenas correio eletrônico e pequenos arquivos.

Em 1990 decide-se pela implantação de uma Rede Nacional de Pesquisa e recomenda-se a adoção da tecnologia TCP/IP. Em 1992 na ECO 92 é instalado o backbone da RNP.

Em julho de 1993, a RNP contava com cerca de 120 endereços IP, de 60 entidades ligadas diretamente à Internet, das quais 20 eram universidades.

Em se tratando do início das operações em 1995, pode-se dizer que foi e está sendo um tanto conturbada. O governo lançou um documento com disposições sobre o acesso, mas logo foram lançadas duas portarias que regulamentam: a Rede Nacional de Pesquisas é a administradora do acesso comercial da Internet, ficando a cargo da Embratel a administração dos meios de comunicação nacionais e internacionais.

A Internet estará à disponível comercialmente a partir de setembro deste ano, no máximo. É o tempo que o governo tem para adequar a RNP para a entrada de empresas privadas no sistema.

A Embratel única empresa autorizada até hoje a fazer esta conexão, deixa de prestar o serviço no final do ano, passando seus clientes para empresas privadas.

A coordenação de endereços (senhas) e a conexão entre sistemas será regulada por um órgão a ser criado, o Comitê Gestor Internet.

O governo só oferecerá o acesso à Internet nas regiões onde a iniciativa privada não se interessar em atuar. Neste caso, o acesso seria feito pelas companhias telefônicas estaduais.

Para o usuário brasileiro ter acesso entre os cerca de 30 milhões no mundo, terá de ficar na fila. Em fevereiro a Embratel estimava em 10 mil cadastrados, no final de abril em 25 mil, agora já estima-se em 50 mil. Há uma estimativa do 
número dos brasileiros que já acessam a Internet que é em torno de 65 mil usuários.

Empresas brasileiras já estão preparando para quando a Internet for oficialmente aberta comercialmente. É o caso do Mappin (MappinNet), onde oferecerão cerca de 1000 itens on-line. No ramo bancário alguns bancos já operam o Home-banking, uma espécie de serviço que sai do Banco e vai direto para o computador da residência do correntista.

O Brasil está na Internet através de dados.

A banda Sepultura, a Xuxa, os grafites das ruas de São Paulo, uma escola de samba. Existe também uma Biblioteca virtual de língua e literatura, onde encontra-se uma quantidade de livros e referências bibliográficas em português.

O LSI/USP (Laboratório de sistemas integráveis da USP) http://www.Isi.usp.br, tem uma das páginas universitárias mais simpáticas. Com uma câmera de vídeo que mostra os usuários em seu trabalho pelas instalações do laboratório.

Um dos serviços brasileiros mais úteis é a companhia de Informações que é gratuita e fica no Instituto de Física da USP, que fornece sinopses de jornais, distribui listas de discussões e centraliza uma série de informações sobre a Internet (fileserv@if.usp.br) c/a frase send news guide.

Outro serviço é o Caminho do Silício (http:/ /crca www.ucsd edu/bobw/brazil.html) sobre música de computador.

O principal grupo sobre o Brasil na Usenet é soc.culture. brazil. Outro ponto de encontro é o canal \#brasil, no IRC (Internet Relay Chart), programa que permite conversa entre vários usuários simultaneamente.

Os órgãos públicos poderão acessar a Internet através do REBIM- Rede Brasileira de Informações Municipais. Permitirá a cada município cadastrado no serviço acesso à informações municipais.

O PT Partido dos Trabalhadores pretende criar até o final do ano. a Agência PT. A intenção é se tornar um provedor (intermediário) do acesso à rede e estabelecer ligação on-line entre todos os comitês do país, além de um serviço de notícias.
No ABC paulista, algumas instituições já estão se plugando na rede. É o caso do Instituto Metodista de Ensino Superior em São Bernardo do Campo, a primeira instituição de ensino a se conectar. Foi criado um BBS (Bulletin Board system), uma espécie de pequeno sistema que permite a troca de mensagens de correio eletrônico além de arquivos, no Hospital Jardim em Santo André, com o intuito de ter o prontuário dos pacientes por até 30 anos.

O Ciesp em Diadema pretende trabalhar com exportação primeiramente com 20 usuários que já exportam seus produtos.

$\mathrm{O}$ associado que quiser navegar pela rede terá que ir até o Ciesp para ser orientado.

\section{EQUIPAMENTOS E PROGRAMAS PARA CONEXÃO}

As empresas estão apostando tudo para entrarem no mercado de hardware e software que servirão de acesso à Internet. É um mercado promissor, já que as estatísticas dizem algo em torno de 100 milhões de usuários em 1999. Internet:

Surgem alguns programas de conexão à

HOTJAVA- programa da Sun Microsystems para criar páginas de World Wide Web. Ele tem recursos para criar animações e objetos em três dimensões. Será lançado com um browser (programa para visualizar páginas da Web). Apenas roda em máquinas da Sun com sistema operacional Solaris.

A Microsoft traz a versão do WINDOWS NT 3.5 com servidor de WWW embutido. Há mais facilidade na configuração para acesso à rede.

O Word 6.0 for windows começa a ser vendido com arquivo para criar HOME PAGE.

A IBM Brasil prepara seu servidor de WWW no RJ.

O programa Show Me Tv da Sun, permite transmissão de vídeo e áudio na rede. Sua vocação é de vídeos para treinamento interno, acesso à TV interativa e Broadcasting.

As máquinas pioneiras são: Sun 
Microsystems(Netra) Digital(Alphaserver), Tandem (servidores Hymalaia).

Estas siglas são conhecidas entre os usuários que navegam pela rede:

FTP - File Transfer Protocol- principal método para transferência de arquivo.

GOPHER - permite que um usuário acesse os diversos recursos de forma dedutiva. Busca simultânea de informações.

PPP -Point to point protocol. Protocolo para conexão usando linha telefônica.

SLIP - Serial line Internet Protocol. Protocolo usado na conexão via linha discada.

TCP/IP - Transmission Control Protocol/ Internet Protocol. Protocolos utilizados para transferência de arquivos, correio eletrônico e login remoto.

FINGER - comando que permite que um usuário possa encontrar outro usuário na rede para conversar. Digite finger e o endereço do destinatário. Dessa forma sabe-se se o interlocutor está conectado e qual o último acesso dele à rede.

TELNET - principal protocolo para criação de uma conexão com um computador remoto.

WAIS - Wide Area Information Server. Conhecido como uma das mais avançadas ferramentas para indexação de dados.

Um programa que está tendo sua chegada comemorada, é o INTERNET PHONE. Significa que se pode falar com alguém do outro lado do mundo em viva voz. pagando valor semelhante ao da tarifa local. Custará cerca de R \$ 50,00. Esta versão permite a conversa entre 2 usuários. Existe uma versão para rede em WINDOWS WORKGROUPS

O Internet Phone tem a versão shareware(de domínio público) que dá direito a 2 minutos de conversa.

O OS/2, novo sistema operacional da IBM, permite acesso direto à Internet: traz ícones animados, possibilita abertura de várias máquinas virtuais.

O MBONE (Multicasting Backbone)- espécie de rede virtual de computadores dentro da rede, com máquinas capazes de retransmitir imagens de vídeo em tempo real.

O IUMA, um arquivo de rock e pop na rede, utiliza regularmente o MBONE para transmitir shows.

vBNS- Very High Speed Backbone network Service. Sistema Operado pela NSF (National Science Foundation) em conjunto com a MCI. Altíssima velocidade (conexões de 155 mbits por segundo-mais de 10 mil vezes a velocidade de transmissão de um modem de 14,4 mil bps).

A NSFNet saiu em abril da Internet e a vBNS entra no mesmo momento. A NSFNet sustentava o principal backbone (estrutura básica para funcionamento da rede americana).

CUSeeMe- soft desenvolvido em caráter experimental pela Univ.de Cornell (EUA) que permite teleconferência.

REALVIDEO- soft que permite transmissão de som em tempo real.

IPHONE- permite conversas telefônicas pela rede. Vantagem maior-economia. É como um telefone internacional quase gratuito.

WEBSPACE- criação de telas em três dimensões.

\section{BBS - BULLETIN BOARD SYSTEM}

O Brasil possui cerca de 200 BBS- centrais eletrônicas de serviços, pagos ou gratuitos, variam de previsões meteorológicas a suporte técnico de empresas de informática.

Funciona mais ou menos como a Internet. É necessário um computador, um telefone e um modem.

Os maiores BBS do país estão cobrando cerca de 10 dólares mensais e dão direito a uma hora diária .

Um dos mais tradicionais BBS do país, o Mandic, mantém por exemplo, um acervo de 7 gbytes repletos de softwares de domínio público.

Dos mais de 100.000 BBS nos EUA, o 
Brasil pode acessar pelo menos uma centena deles. Há vários serviços que são prestados pelo BBS: um deles é o Mandic em que o usuário encontra trabalhos de várias disciplinas na parte de Conferência Educação.

Outra é a consulta sobre o exame da Fuvest. Tudo do manual do candidato encontra-se lá.

Há também um BBS evangélico: Christian Connection que se propõe a trocar informações sobre religião para evitar e detectar distorções em seitas e igrejas.

\section{METODISTA (IMS) NA INTERNET}

O Instituto Metodista de Ensino Superior é a primeira instituição acadêmica do Grande $\mathrm{ABC}$ a se conectar à Internet. Segundo o vicediretor de graduação e pós-graduação Luiz Roberto Alves, o uso da rede será estritamente acadêmico e a princípio dois computadores, um no prédio Lambda e outro no Delta, estarão à disposição dos alunos e professores. Ele esclarece que quer democratizar o acesso com controle, para evitar desperdícios. É aberto à todos.

Um manual de uso da rede está sendo elaborado e os usuários interessados contarão com a orientação de funcionários treinados.

O IMS está conectado via Fundação de Amparo Pesquisa do Estado de São Paulo (FAPESP), à USP e pela ALTERNEX via Instituto Brasileiro de Análises Econômicas e Sociais (IBASE) no RJ.

Para Alves, a primeira forma de conexão fornecerá informações de caráter mais oficial, Já no Ibase, os usuários encontrarão informações sobre Organizações não governamentais.

O IMS se cadastrou junto à Embratel no final do ano passado.

No dia 7 de Junho houve uma demonstração aberta para oficializar o início do acesso à Internet pela Metodista.

A CESP (Companhia Energética de São Paulo) foi oficialmente o primeiro organismo a ser conectado pela Metodista através da rede.

O IMS fez uma assinatura para se conectar de RENPAC 2000 (Rede Nacional de Pacotes).
Mas esta ainda não dá direito a imagens, apenas texto.

O programa de acesso é o QUICK LINK que está instalado no Windows.

Disca-se o n. do Renpac, dá-se o comando Finger que é para escolher o "parceiro de informação" que se quer comunicar. Ao dar esse comando, aparece uma lista com o nome de empresas, instituições e usuários que se poderá conectar a qualquer um deles.

Para que o acesso seja controlado, foi distribuído uma folha cadastral de pesquisadores e priorizadas quatro áreas de pesquisa:

- A cidade e a construção da cidadania - Urbanização (políticas públicas, educação, saúde, cultura, comunicação social, etc.)

- Sociologia no seu sentido mais amplo

- Papel do educador na construção da cidadania

- Ecumenismo, confessionalidade e educação

- O papel da escola confessional na educação

- Diversos movimentos religiosos e suas interligações

- Mercosul

- Desenvolvimento de políticas econômicas e a integração dos países latino-americanos

- Ciência e Tecnologia

- o impacto de novas tecnologias para o desenvolvimento da humanidade

- pesquisas científicas

- ciências biomédicas

\section{LIBERDADE DE USO DA REDE X POLÊMICAS}

Têm surgido várias polêmicas sobre o uso deliberado da Internet, no que diz respeito à sigilo e segurança de dados. Quanto mais computadores estiverem interligados mais se estará suscetível a perda de dados ou até mesmo de arquivos, por pessoas de má fé.

Uma questão é o funcionamento de um banco virtual, iniciativa apresentada pelo First Virtual, banco americano que só funciona na Internet. Os pagamentos por um n. de identificação associado ao cartão de crédito, são autorizados por correio eletrônico. 
Isto leva a crer que é possível várias pessoas descobrirem o n. do cartão de crédito alheio e aí, partirem para o ataque.

Fatos semelhantes aconteceram no Brasil, quando em 17 de abril deste ano, os computadores da Empresa Brasileira de Pesquisas Agropecuárias foram invadidos. $\mathrm{O}$ "cracker" conseguiu apagar arquivos e mandou mensagens ameaçando outras instituições.

Este criminoso obteve conexão à Internet no servidor da Fundação Tropical André Poselo, uma Ong de Campinas-SP, e de lá se conectou via Telnet (terminal remoto) à Embratel, usando a senha do administrador do sistema que garante acesso a todas as informações do sistema.

$O$ recurso mais usado para garantir a segurança de dados é o chamado FIRE-WALL ou parede de fogo. Um bom software corporativo de acesso à rede deve incluí-lo ou oferecer algum dispositivo semelhante.

Isto tudo é causa da liberdade que se tem na rede. É possível deixar e receber mensagens anônimas, incitar ao crime, à violência, ao racismo e não correr qualquer tipo de punição.

Alguns grupos nos EUA começam a se preocupar com isto: polícia, consultores de segurança de grandes companhias e até profissionais liberais.

Isto porque um terço das casas norteamericanas tem computadores e há 10 milhões de usuários da rede.

Começou-se a questionar sobre a segurança na rede, depois do atentado de Oklahoma, quando um simpatizante da extrema direita detonou um prédio do governo federal.

Arquivos consultados em fóruns da Internet, veio à tona a troca de receitas de bombas caseiras. Depois do atentado, a Internet passou a ser veículo de mais ou menos 5000 mensagens diárias referentes a explosivos e bombas.

Grupos que monitoram os radicais da direita americana descobriram a existência de pelo menos oito BBS dedicados ao debate, troca de mensagens, etc.

Em Detroit, há o grupo separatista Resistance Inc. que criou um fórum só para discutir seu objetivo: de criar um país independente só para brancos, e na Flórida um ex-integrante da $\mathrm{Ku}$ Klux Klan formou uma entidade dedicada a disseminar informação racista.

A Internet Society é uma entidade sem fins lucrativos, criado em 1992, que cuida de assuntos ligados à rede, principalmente no que se refere a definições de seus padrões.

A I.Soc. participa ativamente das discussões dos fóruns eletrônicos e pode influenciar no andamento de muitos grupos.

Houve um episódio em que a I.Soc. foi acusada de encerrar um fórum de cunho feminista na rede. O fim deste grupo foi patrocinado pela I.Soc., que teria considerado as discussões "impróprias". A entidade negou ter poder sobre isto, só que vários grupos são fechados, e isto se deve a um centro de poder.

Dois jornalistas tiveram seu telefone bloqueado, bem como não receberam mensagens via Internet, sendo que eles reservavam várias horas do dia para isto.

Alguém enviou respostas depois dizendo que eles deveriam ficar de fora da rede, onde senão (os jornalistas) a transformariam em uma fossa transbordante de ganância.

Sendo assim, resta esperar e crer que a Internet não vai se tornar mais uma arma nas mãos do homem.

\section{BIBLIOGRAFIA}

AMARAL, Paulo. Internet abre campo para negócios. Diário do Grande $A B C$, Santo André, 9 maio 1995. Informática, p.3.

CHAVES para cessar o mundo. Informática Exame, São Paulo, v.10, n.107, p.38-42, fev. 1995.

CHEGAM produtos para a Net. Diário do Grande $A B C$, Santo André, 9 maio 1995. Informática, p.4.

CHIARELLI, Marco. Internet. Super Interessante, São Paulo, v8, n.4, p.39-45, abr. 1994.

COMO obter acesso à maior das redes. Diário do Grande ABC, Santo André, 9 maio 1995. Informática, p.2.

DIAS, Melina. Aluno da Metodista vai acessar a Inf.Inf., Londrina, v.2, n.2, p.13-22, jul./dez. 1997 
Internet. Diário do Grande $A B C$, Santo André, 6 jun. 1995. Informática, p.6.

Evangélico cria primeiro BBS teológico. Diário do Grande $A B C$, Santo André, 23 maio 1995. Informática, p.2.

. Hospital da região adere à multimídia

e cria BBS próprio. Diário do Grande $A B C$, Santo André, 13 jun. 1995. Informática, p.3.

. IMS inaugura conexão à Internet dia

7. Diário do Grande ABC, Santo André, 30 maio 1995. Caderno 1, p.3.

. Internet inicia amanhã operação

comercial no Brasil. Diário do Grande $A B C$,

Santo André, 30 abr. 1995. Caderno A, p.10.

. Programa permite comunicação em

viva voz pela rede Internet. Diário do Grande

$A B C$, Santo André, 30 maio 1995. Informática, p.1.

EMBRATEL. perde controle do acesso à Internet. Folha de São Paulo, São Paulo, 25 abr. 1995. Informática, p.12.

ENTENDA as siglas da megarrede. Diário do Grande $A B C$, Santo André, 9 maio 1995. Informática, p.4.

ERCÍLIA, Maria. Amor e sexo têm variações na Internet. Folha de São Paulo, São Paulo, 7 jun. 1995. Informática, p.9.

. Conheça o Brasil que está na Internet.

Folha de São Paulo, São Paulo, 17 maio 1995. Informática, p.7.

. Contagem regressiva. Folha de São

Paulo, São Paulo, 25 abr. 1995. Ilustrada, Netvox, p.4.

. Inauguração virtual. Folha de São

Paulo, São Paulo, 2 maio 1995. Ilustrada, Netvox, p.4.

. Internet cria conceito de feiras virtuais. Folha de São Paulo, São Paulo, 12 abr. 1995. Informática, p.7.

. Internet do futuro tem cor e vídeo.

Folha de São Paulo, São Paulo, 31 maio 1995. Informática, p.1.

. O jogo de paciência. Folha de São

Paulo, São Paulo, 6 jun. 1995. Ilustrada, Netvox, p.4.

Óleo de serpente. Folha de São Paulo, São Paulo, 30 maio 1995. Ilustrada, Netvox, p.4.

. San Francisco urgente. Folha de São Paulo, São Paulo, 21 maio 1995. Ilustrada, Netvox, p.4.

. Satan chega amanhã. Folha de São Paulo, São Paulo, 4 abr. 1995. Ilustrada, Netvox, p.4.

. A teia vai engolir a rede. Folha de São Paulo, São Paulo, 31 jan. 1995. Ilustrada, Netvox, p.3.
. Uma rota longa demais. Folha de São Paulo, São Paulo, 13 jun. 1995. Ilustrada, Netvox, p.4. . A vingança do telefone. Folha de São Paulo, São Paulo, 18 abr. 1995. Ilustrada, Netvox, p.4.

FONSECA, Tatiana. Bem vindo à Internet. Imprensa, São Paulo, v.8, n.91, p.28-35, abr. 1995.

GOVERNO regulamenta acesso à Internet. Folha de São Paulo, São Paulo, 1 jun. 1995. Caderno 1, p.3.

GRÁFICOS facilitam passeio pela Internet. Folha de São Paulo, São Paulo, 8 fev. 1995. Informática, p.6.

GUERRA fria estimulou criação. Diário do Grande $A B C$, Santo André, 9 maio 1995. Informática, p.4.

INTERNET tem imagens de homem fatiado. Folha de São Paulo, São Paulo, 22 mar. 1995. Informática, p.5.

JAPIASSU, Daniel. Aproveite bem o tempo de ligação com seu BBS. Folha de São Paulo, São Paulo, 31 maio 1995. Informática, p.2.

LAQUEY, Tracy, RYER, Jeanne C. O manual da Internet: um guia introdutório para acesso às redes globais. Tradução por Insight Serviços de Informática. Rio de Janeiro : Campus, 1994. 270p.

LEI e desordem na Internet. PC Magazine Brasil, São Paulo, v.5, n.5, p.14, maio 1995.

MAPPINET vai vender mil itens. Diário do Grande $A B C$, Santo André, 9 maio 1995. Informática, p.3.

MARTINS, Ivan. O que é mesmo a Internet. Exame, São Paulo, v.27, n.11, p.108-114, 24 maio 1995.

MEDIDA leva Internet para o setor privado. Diário do Grande $A B C$, Santo André, 1 jun. 1995. Caderno 1, p.11.

MICHELACCI, Alexandre. Bastidores de Batman vão integrar a Internet. Diário do Grande $A B C$, Santo André, 6. Jun. 1995. Cultura\&Lazer, p.4.

MORAES, Marina. Liberdade da rede causa polêmica nos EUA. Folha de São Paulo, São Paulo, 17 maio 1995. Informática, Linha direta, p.2.

O MUNDO do outro lado da linha. Informática Exame, São Paulo, v.9, n.97, p.64-68, abr. 1994.

O MUNDO sem fronteiras. Informática Exame, São Paulo, v.10, n.109, p.62-68, abr. 1995.

OPERAÇÃO comercial tem início em maio. Folha de São Paulo, São Paulo, 8 fev. 1995. Informática, p.7.

PELISSIER, Denise. Internet: convite a viajar. 
O Correio da Unesco, Rio de Janeiro, v.23, n.4, p.17, abr. 1995.

PEREZ, Luís. BBS traz dicas para vestibulando. Folha de São Paulo, São Paulo, 2 nov. 1994. Informática, p.1.

PLASSE, Marcel. Internet tem fórum de debate sobre a banda Beastie Boys. Folha de São Paulo, São Paulo, 17 abr. 1995. Ilustrada, p.3.

QUANTO custa usar a Internet. Folha de São Paulo, São Paulo, 16 fev. 1995. Turismo, p.21.

REDE ligará Internet a prefeituras de todo o país neste ano. Diário do Grande $A B C$, Santo André, 23 maio 1995. Informática, p.2.

REGGIANI, Lúcia. Namoro virtual. Folha de São Paulo, São Paulo, 7 jun. 1995. Informática, p.1.

SARAIVA JÚNIOR, Jacilio. Mundo afora. $P C$ World, Rio de Janeiro, v.4, n.36, p.8-22, jun. 1995.

SILVESTRE JÚNIOR, Paulo Fernando. A rede de influência na Internet. Folha de São Paulo, São Paulo, 22 jan. 1995. Caderno Mais, Ciência, p.16.

SIQUEIRA, Rodrigo. Laboratório químico alemão entra em rede. Folha de São Paulo, São Paulo, 26 abr. 1995. Informática, p.8.

SISTEMA transmite em alta velocidade. Folha de São Paulo, São Paulo, 31 maio 1995. Informática, p.3.

SQUIRRA, Sebastião. Entenda o endereço Internet. Diário do Grande $A B C$, Santo André, 7 fev. 1995. Informática, p.2. . Internet à sua disposição. Diário do Grande ABC, Santo André, 24 jan. 1995. Informática, p.2.

TRANSMISSÃO de vídeo ainda é experimental. Folha de São Paulo, São Paulo, 31 maio 1995. Informática, p.3.

TRIBO Internet. PC World, Rio de Janeiro, v.4, n.35, p.58-59, maio 1995.

USUÁRIO brasileiro tem poucos recursos. Folha de São Paulo, São Paulo, 31 maio 1995. Informática, p.11.

USUÁRIOS conversam pela rede. Folha de São Paulo, São Paulo, 31 maio 1995. Informática, p.3.

ZILVETI, Marijô. Internet leva turista numa viagem virtual. Folha de São Paulo, São Paulo, 16 fev. 1995. Turismo, p.21.

. Ligue seu micro à Internet. Folha de São Paulo, São Paulo, 8 fev. 1995. Informática, p.1.

\section{Maristela Dourado Rocha}

Bibliotecária de referência do Instituto Metodista de Ensino Superior - Faculdade de Teologia São Bernardo do Campo - São Paulo.

\section{Title}

The Metodist connection to Internet: the largest computer netword in the planet.

\section{Abstract}

This work is a survey about the Internet: the computer network that links a computer to others, located in a ny part of the world, aiming at exchanging information, knowledge, and files. The work also deals with the acess to Internet obtained by Instituto Metodista de Ensino Superior, the first academic institution in Grande $\mathrm{ABC}$ to be worldwidely connected.

\section{Keywords}

Internet - Computer network - Automatic Data Processing

Apresentado no COBIBiii em agosto de 1995 